\title{
Recombination mechanism of photoluminescence in InN epilayers
}

\author{
S.P. Fu ${ }^{\text {a }}$, Y.F. Chen ${ }^{a}{ }^{*}$, Keewee Tan ${ }^{b}$ \\ ${ }^{a}$ Department of Physics, National Taiwan University, Taipei 106, Taiwan, ROC \\ ${ }^{\mathrm{b}}$ School of Applied and Engineering Physics, Cornell University, Ithaca, NY 14853, USA
}

Received 8 August 2005; received in revised form 5 November 2005; accepted 8 November 2005 by A. Pinczuk

Available online 21 November 2005

\begin{abstract}
We report an investigation of the recombination mechanism for photoluminescence (PL) in InN epilayers grown by molecular beam epitaxy and metal-organic chemical vapor deposition with a wide range of free electron concentrations from $3.5 \times 10^{17}-5 \times 10^{19} \mathrm{~cm}^{-3}$. We found that the PL spectra are strongly blueshifted with increasing excitation intensity. For all the samples studied, the exponent of the relationship between the integrated PL intensity and the excitation intensity is very close to unity and independent of the temperature. By assuming Gaussian fluctuations of the random impurity potential, calculation based on the 'free-to-bound' recombination model can be used to interpret our results very well and it correctly reproduces the development of the total PL peak shift as a function of carrier concentration. It is concluded that the PL transition mechanism in InN epifilms can be characterized as the recombination of free electrons in the conduction band to nonequilibrium holes in the valence band tail.
\end{abstract}

(C) 2005 Elsevier Ltd. All rights reserved.

PACS: 78.55.Cr; 78.66.Fd

Keywords: D. Recombination mechanism; E. Photoluminescence

\section{Introduction}

InN, the least studied Group-III nitride, has attracted intense interests because of its distinctive physical properties [1-3]. Early reports using infrared absorption technology have shown that the band gap energy of $\mathrm{InN}$ is $1.8-2.0 \mathrm{eV}$ [4] Recently, numerous studies have observed instead a strong PL emission at around $0.7-0.8 \mathrm{eV}$, leading to the revision of the fundamental band gap of InN from $1.8-2.0$ to $0.7-0.8 \mathrm{eV}$ [5-7]. This newly discovered discrepancy has spurred numerous attempts to incorporate $\mathrm{InN}$ in various optoelectronic devices. For instance, the entire visible spectrum can now be spanned by a single material system; therefore, $\mathrm{InN}$ and its alloys have also emerged as promising materials for optoelectronic devices such as light-emitting diodes and solar cells. In addition, the band gap energy of InN makes it suitable for application in $1.55 \mu \mathrm{m}$-telecommunications.

In order to effect the application in optoelectronic devices, it is essential to have a detailed understanding of the optical properties

\footnotetext{
* Corresponding author. Tel.: +8862 33665125; fax: +886223639984.

E-mail address: yfchen@ phys.ntu.edu.tw (Y.F. Chen).
}

0038-1098/\$ - see front matter (C) 2005 Elsevier Ltd. All rights reserved. doi:10.1016/j.ssc.2005.11.013 of InN thin films. Recently, Arnaudov et al. have made an important breakthrough and they reported that the mechanism responsible for the emission at $0.7-0.8 \mathrm{eV}$ can be very well described a model of 'free-to-bound' radiative recombination of degenerate electrons from the conduction band with nonequilibrium holes located in the valence band tails [8]. This model, in principle, resolves the question about the recombination mechanisms in the near band edge region. However, recent low temperature absorption measurements led Shubina et al. to propose that the IR emission at around $0.7 \mathrm{eV}$ may be due to the analogous Mie scattering produced by In clusters [9]. Very recently, Guo et al. have observed a visible luminescence from $\mathrm{InN}$ thin films grown by reactive radio frequency magnetron sputtering and inferred that the emission at $0.7-0.8 \mathrm{eV}$ could be due to In clusters or oxygen-related defects [10]. In addition, Butcher et al., Alexandrov et al. and Intartaglia et al. have also shown that the emissions around $0.7-1.0 \mathrm{eV}$ were consistent with the presence of $0.7 \mathrm{eV}$ deep level trap and/or the recombination of electron-hole pairs or excitons to extrinsic defects or topological defects [11-14]. Thus, the luminescence mechanism(s) of InN are still discordant. As a consequence, further investigation of the optical properties of this material has to be undertaken to clarify the inconsistency.

In this paper, we present a study of the PL recombination mechanism of InN epilayers with a wide range of free electron 
concentrations $\left(n=3.5 \times 10^{17} \mathrm{~cm}^{-3}\right.$ to $\left.n=5 \times 10^{19} \mathrm{~cm}^{-3}\right)$ grown by MBE and MOCVD. We found that the PL spectra are blueshifted with increasing excitation intensity. The exponent of the relationship between the integrated PL intensity and the excitation intensity does not depend on temperature and has a value close to unity. By assuming Gaussian fluctuations induced by random impurity potential, calculation based on the 'free-to-bound' recombination model can be used to interpret our results very well. In addition, the development of the total PL peak shift (defined as the difference of the PL peak energy obtained from the highest and the lowest excitation intensity) as a function of free electron concentration can also be well characterized by the same model. We thus conclude that the 'free-to-bound' recombination model is the underlying mechanism responsible for the PL recombination in InN epifilms.

\section{Experiment}

InN films were grown on (0001) sapphire (sample set I and III) and (111) silicon (sample set II) substrates with AlN or $\mathrm{GaN}$ (sample set I and III) and $\beta-\mathrm{Si}_{3} \mathrm{~N}_{4} / \mathrm{AlN}$ (0001) (sample set II) buffer layers by MBE (sample set I and II) and MOCVD (sample set III). The thickness of the buffer layer ranges from 70 to $300 \mathrm{~nm}$. The InN layer thickness is between $250 \mathrm{~nm}$ and $7.5 \mu \mathrm{m}$, which implies that all the InN films were fully relaxed. Although most of the samples were not intentionally doped, free-electron concentrations ranging from $3.5 \times 10^{17}$ to $5.5 \times$ $10^{18} \mathrm{~cm}^{-3}$ (sample set I), $1 \times 10^{19} \mathrm{~cm}^{-3}$ (sample set II and III) have been found in these samples by Hall effect measurements. Higher free-electron concentrations were achieved by doping InN with Si. The free-electron concentration of these doped samples varies between $1.0 \times 10^{19}$ and $5.0 \times 10^{19} \mathrm{~cm}^{-3}$. The details of the growth process have been published elsewhere [15-17]. X-ray diffraction studies show that these InN epitaxial layers have a high quality and a wurtzite structure with their $c$-axis perpendicular to the substrate surface. Hall mobilities range from several hundred to $2050 \mathrm{~cm}^{2} / \mathrm{V} \mathrm{s}$.

The PL spectra were dispersed by a Spectra Pro $275 \mathrm{i}$ monochromator, and detected by an EOS extended InGaAs detector which has a cut off wavelength of $2.4 \mu \mathrm{m}$. A semiconductor diode laser working at $808 \mathrm{~nm}$ was used as the excitation source. The excitation intensity was varied from $3.9 \times 10^{-4}$ to $3.9 \mathrm{~W} / \mathrm{cm}^{2}$. All the measurements were carried out at $20 \mathrm{~K}$.

\section{Results and discussion}

Typical PL spectra observed under different excitation intensities are shown in Fig. 1(a)-(d). Clearly, the PL spectrum with higher free electron concentration peaked at a higher energy position. Such behavior can be well described by the combination of the Kane's model, Burstein-Moss effect, band tailing effect, and band renormalization effect as shown in the previous reports $[18,19]$. The analysis of the influence of carrier density on PL spectra is, therefore, not the goal of this paper. Instead, we would like to focus on the origin of the
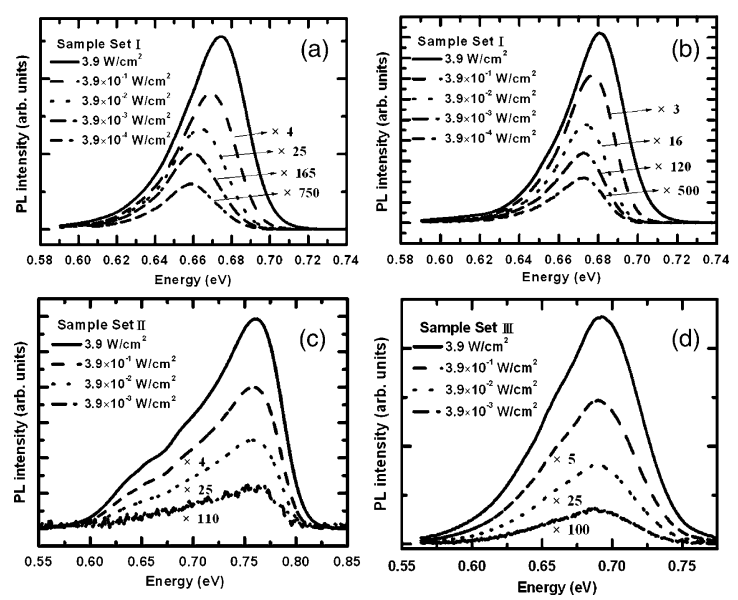

Fig. 1. Photoluminescence spectra of InN samples under different excitation intensities. (a) Sample set I, $n=5.7 \times 10^{17} \mathrm{~cm}^{-3}$, (b) sample set I, $n=1.425 \times$ $10^{18} \mathrm{~cm}^{-3}$, (c) sample set II, $n=9 \times 10^{18} \mathrm{~cm}^{-3}$, (d) sample set III, $n=1 \times$ $10^{19} \mathrm{~cm}^{-3}$.

recombination mechanism of this material, which is still inconclusive at the present stage. Second, the PL peak energies shift to higher energy rapidly with increasing excitation intensity for the low degenerate sample as shown in Fig. 1(a). This result strongly indicates that the recombination mechanism at issue is not 'direct band to band' transition as reported previously [16]. Comparing the PL spectra for the higher degenerate samples shown in Fig. 1(b)-(d) to the previous report, it is clear that the discrepancy may be due to the fact that the residual carrier concentration of those measured samples were too high for the determination of the exact recombination mechanism for pure InN films [16].

Fig. 2 shows the integrated PL intensity on the logarithmic scale as a function of excitation intensity for sample sets I, II, and III. The relationship can be expressed as:

$I_{\mathrm{PL}}=\eta I_{\mathrm{EX}}^{\alpha}$

where $\eta$ is a coefficient which depends on temperature, $I_{\mathrm{PL}}$ is the integrated PL intensity and $I_{\mathrm{EX}}$ is the intensity of the excited source [20,21]. It is well known that the exponent, $\alpha$, strongly depends on the radiative recombination mechanism

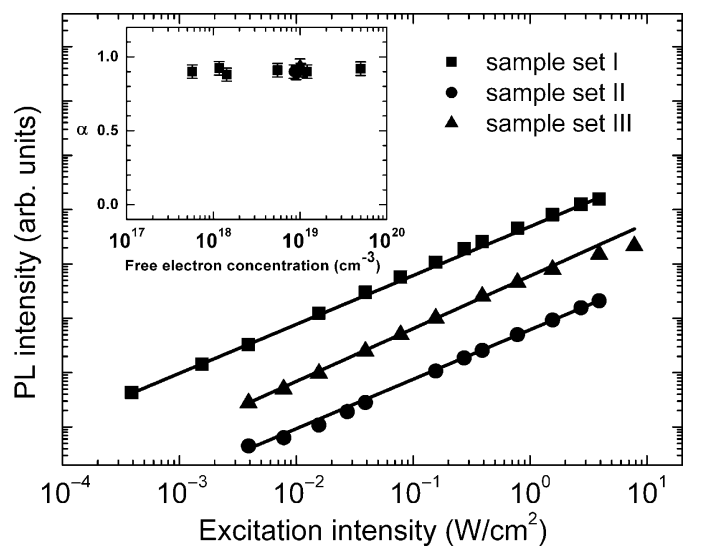

Fig. 2. Integrated photoluminescence intensity as a function of excitation intensity. The theoretical fitting using Eq. (1) is shown as the solid lines. Inset shows $\alpha$ as a function of free electron concentration for all studied samples. 
responsible for the PL signal. For exciton or defect like recombination, the PL intensity is linearly proportional to the excitation intensity $(\alpha=1)$, while for free electron-hole pair recombination the PL intensity is proportional to the square of excitation intensity $(\alpha=2)$ [21]. It can be seen that Eq. (1) can be used to characterize our result very well, and the exponents obtained for all our samples are very close to (but less than) unity as shown in Fig. 2. A possible reason for the slight deviation from unity is that the optical dissipation may be increased with increasing excitation power, which is mainly due to the increase in the diffusion of light and the nonradiative carrier loss with excitation. In addition, we also found that the exponent $\alpha$ does not depend on temperature (not shown).

To further explore the exact recombination mechanism, let us estimate the binding energy of free excitons of InN films. The obtained value is $\sim 2-3 \mathrm{meV}$ with the dielectric constant $\varepsilon=14.61$, the effective electron mass $m_{\mathrm{e}}^{*}=0.05 m_{0}$, and the effective hole mass $m_{\mathrm{h}}^{*}=0.3 m_{0}[8,22,23]$. The localization energy of bound excitons also needs to be taken into account. In general, the values of the localization energy of bound excitons are several millielectron volts for InX compounds $(\mathrm{X}=\mathrm{P}, \mathrm{As}$, and $\mathrm{Sb}$ ), 6-12 $\mathrm{meV}$ for $\mathrm{GaN}$, and $16 \mathrm{meV}$ for $\mathrm{AlN}$ [24-30]. Therefore, the binding energy of bound excitons with respect to the free electron-hole pairs has a value of $20 \mathrm{meV}$ at most. In this case, most excitons of the measured samples will be dissociated at room temperature, and the exponent $\alpha$ will be sensitive to temperature and will increase with increasing temperature if one assumes that the excitonic transition dominates the observed PL spectra. However, this is inconsistent with our experimental result. Therefore, the excitonic recombination can be excluded as well. The most probable candidate for the recombination mechanism may now be defect-related recombination.

For the defect-related recombination mechanism, we consider the 'free-electron-to-bound' recombination (FEBR) model in this heavily doped semiconductor. In this model, the relaxation of momentum conservation for the optical transition enables all electrons distributed up to the Fermi level to participate in the recombination [8,31]. The development of a density-of-states tail in the energy gap due to inhomogeneous impurity distribution and potential fluctuations in heavily doped semiconductors becomes more important for the radiative recombination process with increasing free electron concentration. The localized states in such a band tail can be treated as acceptor like centers distributed above the top of the valence band. This band tail can be expressed as $[8,31]$ :

$G=2 \sqrt{\pi} \frac{4 \pi e^{2}}{\varepsilon R_{\mathrm{s}}}\left(N_{\mathrm{i}} R_{\mathrm{s}}^{3}\right)^{1 / 2}$

where $\varepsilon=14.61$ is the static dielectric constant, $N_{\mathrm{i}}$ is the total ionized impurity concentration, which is assumed to have the same value as the free electron concentration. The ThomasFermi screening length in Eq. (2) is expressed as [8,31]:

$R_{\mathrm{s}} \cong \frac{a_{\mathrm{B}}}{2}\left(n a_{\mathrm{B}}^{3}\right)^{-1 / 6}$
The effective Bohr radius of electrons in Eq. (3) is expressed as $[8,31]$ :

$a_{\mathrm{B}}=\frac{\varepsilon}{4 \pi e^{2}} \frac{h^{2}}{4 \pi^{2} m^{*}}$

The nonequilibrium holes were thus localized in the potential minima of the valence band tails near the thermalequilibrium level, which can be expressed as [8]:

$G^{*}=-E_{\mathrm{v}}+\sqrt{2} E_{\mathrm{T}}-\frac{k T}{2}$

This level acts as the quasi-Fermi level in the nonquasiequilibrium recombination FEBR model, which will dominate the observed blueshift as the pumping power increases. This behavior is quite different from that of previous reports, in which the blueshift of the PL peak energy is attributed to the increase of the quasi-electron Fermi level in the conduction band [32]. The expression of the emission intensity $I(h v)$ is given by:

$$
\begin{aligned}
I(h \nu)= & \int_{0}^{\infty} \int_{0}^{\infty} g_{\mathrm{n}}\left(E_{\mathrm{n}}\right) f_{\mathrm{n}}\left(E_{\mathrm{n}}\right) g_{\mathrm{p}}\left(E_{\mathrm{p}}\right) f_{\mathrm{p}}\left(E_{\mathrm{p}}\right) \\
& \times \delta\left(E_{\mathrm{n}}-E_{\mathrm{p}}-E_{\mathrm{g}}-h \nu\right) \mathrm{d} E_{\mathrm{n}} \mathrm{d} E_{\mathrm{p}}
\end{aligned}
$$

Here, $g_{\mathrm{n}}\left(E_{\mathrm{n}}\right)$ and $g_{\mathrm{p}}\left(E_{\mathrm{p}}\right)$ are the density of states in the conduction and valence band at the electron and hole energies $E_{\mathrm{n}}$ and $E_{\mathrm{p}}$, respectively, $f_{\mathrm{n}}$ and $f_{\mathrm{p}}$ are their nonequilibrium Fermi-Dirac functions. By assuming Gaussian fluctuations of the random impurity potential, the density of states in Eq. (6) are replaced by

$g_{\mathrm{s}}\left(E_{\mathrm{p}}\right)=g_{\mathrm{p}}\left(E_{\mathrm{p}}\right)\left(\frac{G}{E_{\mathrm{p}}}\right) G_{0}\left(\frac{E_{\mathrm{p}}}{G}\right)$

Here, $G_{0}$ is given by:

$G_{0}(x)=\pi^{-0.5} \int_{-\infty}^{x} e^{-y^{2}}(x-y)^{0.5} \mathrm{~d} y$

where $x$ is the dimensionless energy $x=E_{\mathrm{p}} / G$. It should be noted that the energy position of the emission band is mainly determined by the term $g_{\mathrm{p}}\left(E_{\mathrm{p}}\right) f_{\mathrm{p}}\left(E_{\mathrm{p}}\right)$ in Eq. (6). As a consequence, one could hypothesize that the PL intensities in this case are proportional to the hole concentration in the valence band tail:

$I_{\mathrm{PL}} \propto \int_{0}^{\Delta E} g_{\mathrm{s}}\left(E_{\mathrm{p}}\right) f_{\mathrm{p}}\left(E_{\mathrm{p}}\right) \mathrm{d} E_{\mathrm{p}}$

In this paper, the nonequilibrium carrier density generated by the external excitation is much lower than the doping concentration. For example, for the maximum excitation intensity of $3.9 \mathrm{~W} / \mathrm{cm}^{-2}$ used here, it corresponds to a generation of carrier density of about $10^{16} \mathrm{~cm}^{-3}$ [32]. Thus, the PL peak shift due to the increase of quasi-electron Fermi level in the conduction band with increasing excitation 


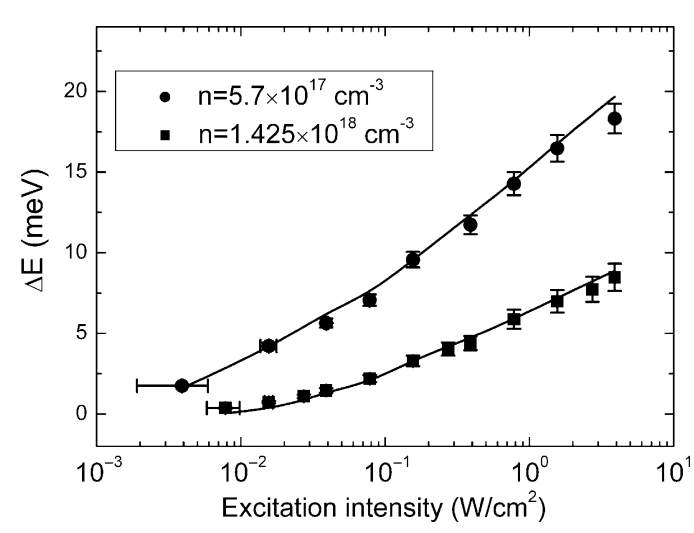

Fig. 3. $\Delta E$ as a function of excitation intensity (sample set I). Calculations using Eqs. (1) and (9) are shown as the solid lines.

intensity can be excluded, which is quite different from the report recently [32]. The PL peak shifts $(\Delta E \mathrm{~s})$ from the lowest measured value as a function of the excitation intensity for the InN samples with free electron concentration of $5.7 \times 10^{17}$ and $1.425 \times 10^{18} \mathrm{~cm}^{-3}$ are shown in Fig. 3. The calculations using Eqs. (1) and (9) are also shown in Fig. 3 as the solid lines. We found that Eqs. (1) and (9) can be used to describe the PL peak shift very well.

Fig. 4 shows the total blueshift PL peak energy $\Delta E_{\mathrm{T}}$ (defined as the difference of the PL peak energy obtained from the highest and the lowest excitation intensity) for all our samples. We found that $\Delta E_{\mathrm{T}}$ strongly depends on the free electron concentration, which varies as much as $17 \mathrm{meV}$ at low carrier concentration and as little as $\sim 0 \mathrm{meV}$ at high carrier concentration. Intuitively, this behavior can be easily understood because the density of tail states induced by impurities is larger for the sample with higher carrier concentration. Thus, it is difficult to change the quasi-Fermi level of the nonequilibrium holes. As further justification of our hypothesis, it is found that calculations using Eqs. (1) and (9) can also characterize the experimental result very well, as shown in Fig. 4. We thus conclude that the PL recombination originates from the free electrons in the conduction band to nonequilibrium holes located in the valence band tail. Our results

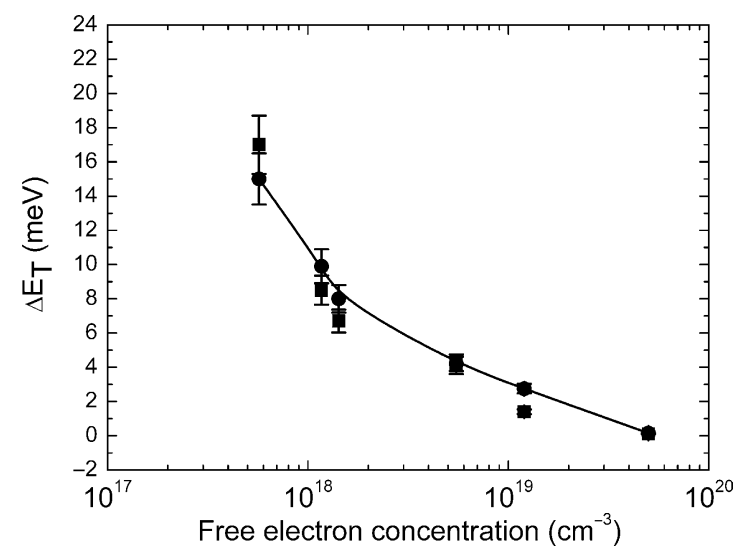

Fig. 4. $\Delta E_{\mathrm{T}}$ vs free electron concentration (shown as solid squares). Calculations using Eqs. (1) and (9) are shown as solid circles. The solid line is the guide for the eyes. shown here seem inconsistent with the literature reported recently. For these reports, the origin of the $0.7-0.8 \mathrm{eV}$ emission is attributed to the extrinsic defects [11-13,32]. However, they are in agreement with the fact that the volume fraction of oxide precipitates in the epitaxial $\mathrm{InN}$ is much too low to significantly affect their optical absorption and reflection spectra, and the recombination mechanism(s) is/are, therefore, not associated with the extrinsic defects [33].

\section{Conclusion}

In conclusion, we have reported a detailed study of the recombination mechanism responsible for the photoluminescence in InN films with a wide range of free electron concentrations from $3.5 \times 10^{17}$ to $5 \times 10^{19} \mathrm{~cm}^{-3}$. We found that the PL spectra are strongly blueshifted with increasing excitation intensity in the sample with low carrier concentrations. The exponent of the relationship between the PL intensity and the excitation intensity is very close to unity, and it does not depend on temperature. By assuming Gaussian fluctuations of random impurity potential, calculation based on the 'free-to-bound' recombination model can be used to describe our results very well. The recombination mechanism in InN films, therefore, can be characterized as free electrons in the conduction band to nonequilibrium holes in the valence band tail.

\section{Acknowledgements}

We like to thank Dr W. Schaff of Cornell University, Dr S. Gwo, and Dr C.A. Chang for providing the samples studied. This work was supported by the National Science Council and Ministry of Education of the Republic of China.

\section{References}

[1] E. Bellotti, B.K. Doshi, K.F. Brennan, J.D. Albrecht, P.P. Ruden, J. Appl. Phys. 85 (1999) 916.

[2] B.E. Foutz, S.K. O’Leary, M.S. Shur, L.F. Eastman, J. Appl. Phys. 85 (1999) 7727.

[3] S.K. O'Leary, B.E. Foutz, M.S. Shur, U.V. Bhapkar, L.F. Eastman, J. Appl. Phys. 83 (1997) 826.

[4] T.L. Tansley, C.P. Foley, J. Appl. Phys. 59 (1986) 3241.

[5] V.Yu. Davydov, A.A. Klochikhin, R.P. Seisyan, V.V. Emtsev, S.V. Ivanov, F. Bechstedt, J. Furthmuller, H. Harima, A.V. Mudryi, J. Aderhold, O. Semchinova, J. Graul, Phys. Status Solidi B 229 (2002) R1.

[6] J. Wu, W. Walukiewicz, K.M. Yu, J.W. Ager III, E.E. Haller, H. Lu, W.J. Schaff, Y. Saito, Y. Nanishi, Appl. Phys. Lett. 80 (2002) 3967.

[7] T. Matsuoka, H. Okamoto, M. Nakao, H. Harima, E. Kurimoto, Appl. Phys. Lett. 81 (2002) 1246.

[8] B. Arnaudov, T. Paskova, P.P. Paskov, B. Magnusson, E. Valcheva, B. Monemar, H. Lu, W.J. Schaff, H. Amano, I. Akasaki, Phys. Rev. B 69 (2004) 115216.

[9] T.V. Shubina, S.V. Ivanov, V.N. Jmerik, D.D. Solnyshkov, V.A. Vekshin, P.S. Kop'ev, A. Vasson, J. Leymarie, A. Kavokin, H. Amano, K. Shimono, A. Kasic, B. Monemar, Phys. Rev. Lett. 92 (2004) 117407.

[10] Q.X. Guo, T. Tanaka, M. Nishio, H. Ogawa, X.D. Pu, W.Z. Shen, Appl. Phys. Lett. 86 (2005) 231913. 
[11] K.S.A. Butcher, Preface for the Proceedings of the First International Indium Nitride Workshop, Fremantle, Australia, 16-20 November 2003 [J. Cryst. Growth 269 (2004) vii ].

[12] K.S.A. Butcher, in: Q.X. Guo (Ed.), Advanced Materials in Electronics, Research Signpost, Kerala, 2004.

[13] D. Alexandrov, K.S.A. Butcher, M. Wintrebert-Fouquet, J. Cryst. Growth 269 (2004) 77.

[14] R. Intartaglia, B. Maleyre, S. Ruffenach, O. Briot, T. Taliercio, B. Gil, Appl. Phys. Lett. 86 (2005) 142104.

[15] H. Lu, W.J. Schaff, J. Hwang, H. Wu, G. Koley, L.F. Eastman, Appl. Phys. Lett. 79 (2001) 1489.

[16] S. Gwo, C.-L. Wu, C.-H. Shen, W.-H. Chang, T.M. Hsu, J.-S. Wang, J.-T. Hsu, Appl. Phys. Lett. 84 (2004) 3765.

[17] C.A. Chang, C.F. Shih, N.C. Chen, P.H. Chang, K.S. Liu, Phys. Status Solidi C 1 (2004) 2559.

[18] J. Wu, W. Walukiewicz, S.X. Li, R. Armitage, J.C. Ho, E.R. Weber, E.E. Haller, H. Lu, W.J. Schaff, A. Barcz, R. Jakiela, Appl. Phys. Lett. 84 (2004) 2805.

[19] S.P. Fu, T.T. Chen, Y.F. Chen, submitted for publication.

[20] S. Jin, Y. Zheng, A. Li, J. Appl. Phys. 82 (1997) 3870.

[21] Q. Liu, S. Derksen, A. Lindner, F. Scheffer, W. Prost, F.J. Tegude, J. Appl. Phys. 77 (1995) 1154.
[22] F. Bernardini, V. Fiorentini, D. Vanderbilt, Phys. Rev. Lett. 79 (1997) 3958.

[23] S.P. Fu, Y.F. Chen, Appl. Phys. Lett. 85 (2004) 1523.

[24] J. Kang, F. Matsumoto, T. Fukuda, J. Appl. Phys. 81 (1997) 905.

[25] T.S. Rao, C. Lacelle, R. Benzaquen, S.J. Rolfe, S. Charbonneau, P.D. Berger, A.P. Roth, T. Steiner, M.L.W. Thewalt, J. Appl. Phys 76 (1994) 5300.

[26] Y. Lacroix, S.P. Watkins, C.A. Tran, M.L.W. Thewalt, Appl. Phys. Lett. 66 (1995) 1101

[27] J.A.H. Stotz, M.L.W. Thewalt, Phys. Rev. B 67 (2003) 155210.

[28] G. Pozina, J.P. Bergman, T. Paskova, B. Monemar, Appl. Phys. Lett. 75 (1999) 4124.

[29] V. Kirilyuk, P.R. Hageman, P.C.M. Christianen, P.K. Larsen, M. Zielinski, Appl. Phys. Lett. 79 (2001) 4109.

[30] K.B. Nam, J. Li, M.L. Nakarmi, J.Y. Lin, H.X. Jiang, Appl. Phys. Lett. 82 (2003) 1694.

[31] B. Arnaudov, T. Paskova, E.M. Goldys, S. Evtimova, B. Monemar, Phys. Rev. B 64 (2001) 045213.

[32] F. Chen, A.N. Cartwright, H. Lu, W.J. Schaff, Physica E 20 (2004) 308.

[33] X. Xu, P. Specht, R. Armitage, J.C. Ho, E.R. Weber, C. Kisielowski, Appl. Phys. Lett. 87 (2005) 092102. 